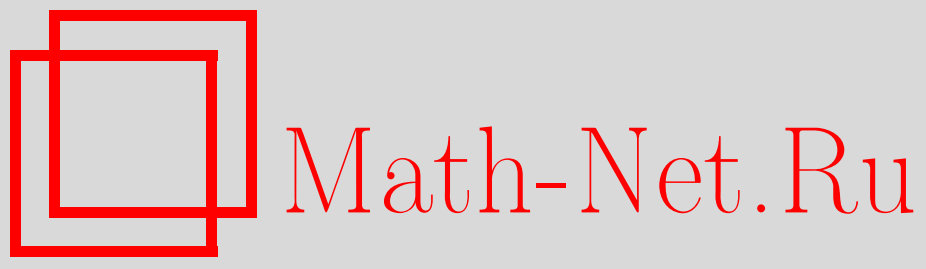

Ю. М. Березанский, Обобщенная проблема моментов, связанная с корреляционными мерами, Функи. анализ и его прил., 2003, том 37, выпуск 4, 86-91

DOI: https://doi.org/10.4213/faa170

Использование Общероссийского математического портала MathNet.Ru подразумевает, что вы прочитали и согласны с пользовательским соглашением

http://www.mathnet.ru/rus/agreement

Параметры загрузки:

IP : 54.162 .27 .143

26 апреля 2023 г., 16:57:40

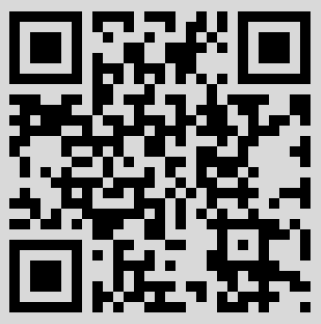




\title{
Обобщенная проблема моментов, связанная с корреляционными мерами*
}

\author{
(c) 2003. Ю. М. БЕРЕЗАНСКИЙ
}

\section{И. М. Гельфанду - с благодарностью}

Хорошо известно, что степенная проблема моментов, включая ее бесконечномерный аналог, тесно связана со спектральными представлениями позитивных функционалов в некоторых коммутативных алгебрах (см., например, [1]). В [2] была введена свертка, позволившая авторам описать меры на пространстве конечных конфигураций, являющиеся корреляционными для мер на пространстве бесконечных конфигураций. В [3] эти результаты были продолжены с помощью техники получения спектральных представлений в соответствующей коммутативной алгебре; их можно было воспринимать как изучение некоторой проблемы моментов.

В этой заметке, продолжающей работу [3], получены и исследованы спектральные представления в более общей ситуации, когда роль корреляционных мер играют соответствующие функционалы. Методы основаны на разложении по обобщенным собственным векторам самосопряженного оператора и восходят к [4-7]. Здесь существенную роль играет также $K$-преобразование, введенное в [8] и изученное в $[8,2]$.

1. Пусть $X-$ связное ориентированное $C^{\infty}$-риманово некомпактное многообразие, $\mathscr{D}=C_{\mathrm{fin}}^{\infty}(X)$ - пространство действительных бесконечно дифференцируемых финитных функций на $X$ с классической топологией, $\mathscr{D}_{c}-$ его комплексификация. Положим $\mathscr{F}_{n}(\mathscr{D}):=\mathscr{D}_{c}^{\widehat{\otimes} n}, n \in \mathbb{N}_{0}=\{0,1, \ldots\}\left(\mathscr{F}_{0}(\mathscr{D})=\mathbb{C}\right)$, где $\widehat{\otimes} n-$ симметрическая тензорная степень, и введем топологическую прямую сумму этих пространств:

$$
\mathscr{F}_{\text {fin }}(\mathscr{D}):=\bigoplus_{n=0}^{\infty} \mathscr{F}_{n}(\mathscr{D}) .
$$

Векторы $f$ «фоковского» пространства (1) имеют вид финитных последовательностей $f=\left(f_{n}\right)_{n=0}^{\infty}, f_{n} \in \mathscr{F}_{n}(\mathscr{D})$; их сходимость - равномерно финитная со сходимостью каждой координаты в соответствующем $\mathscr{F}_{n}(\mathscr{D})$. Такая топология превращает $\mathscr{F}_{\text {fin }}(\mathscr{D})$ в ядерное пространство.

Нам будет необходимо интерпретировать векторы из (1) как функции на пространстве $\ddot{\Gamma}_{X, 0}$ кратных конфигураций. Под такой конфигурацией порядка $n \in \mathbb{N}$ понимается множество $\xi_{n}=\left[x_{1}, \ldots, x_{n}\right]$ из $n$ точек $x_{j} \in X$ (среди них могут быть одинаковые). Иными словами, совокупность $\ddot{\Gamma}_{X}^{(n)}$ всех таких конфигураций равна факторпространству $X^{n} / S_{n}$, где $S_{n}-$ группа перестановок $n$ точек; топология в $\ddot{\Gamma}_{X}^{(n)}$ индуцируется топологией пространства $X^{n}$. Аналогично $(1)$ 
образуем дизъюнктную сумму топологических пространств $\ddot{\Gamma}_{X}^{(n)}$ :

$$
\ddot{\Gamma}_{X, 0}:=\bigsqcup_{n=0}^{\infty} \ddot{\Gamma}_{X}^{(n)}, \quad \ddot{\Gamma}_{X}^{(0)}=\varnothing .
$$

Bектор $f=\left(f_{n}\right)_{n=0}^{\infty}$ из (1) можно интерпретировать как некоторую функцию на $\ddot{\Gamma}_{X, 0}$ : в самом деле, $f_{n}$ принадлежит $\mathscr{D}_{c}^{\widehat{\otimes} n}$ для $n \in \mathbb{N}$ и является симметрической, гладкой и финитной функцией $f\left(x_{1}, \ldots, x_{n}\right)$ на $X^{n}$; поэтому можно положить $f\left(\xi_{n}\right)=f\left(\left[x_{1}, \ldots, x_{n}\right]\right)=f\left(x_{1}, \ldots, x_{n}\right)\left(f_{0} \in \mathbb{C}\right.$ есть функция одной точки $\left.\varnothing\right)$. В результате весь вектор $f$ интерпретируется как (финитная по $n$ ) функция на $\ddot{\Gamma}_{X, 0}$. Мы не будем различать вектор $f \in \mathscr{F}_{\text {fin }}(\mathscr{D})$ и соответствующую функцию $f(\xi), \xi \in \ddot{\Gamma}_{X, 0}$.

Для любого замкнутого множества $\Lambda \subset X$ через $\ddot{\Gamma}_{\Lambda}^{(n)}$ и $\ddot{\Gamma}_{\Lambda, 0}$ будут обозначаться введенные выше пространства с заменой $X$ на $\Lambda$.

2. Превратим пространство $\mathscr{F}_{\text {fin }}(\mathscr{D})$ в коммутативную алгебру $\mathscr{A}(\mathscr{D})$, введя для любых $f, g \in \mathscr{F}_{\text {fin }}(\mathscr{D})$ умножение $\star[2,3]$ :

$$
(f \star g)(\xi)=\sum_{\xi^{\prime} \cup \xi^{\prime \prime} \cup \xi^{\prime \prime \prime}=\xi} f\left(\xi^{\prime} \cup \xi^{\prime \prime}\right) g\left(\xi^{\prime \prime} \cup \xi^{\prime \prime \prime}\right), \quad \xi \in \ddot{\Gamma}_{X, 0},
$$

где суммирование происходит по всем разбиениям конфигурации $\xi$ в объединение непересекающихся конфигураций $\xi^{\prime}, \xi^{\prime \prime}, \xi^{\prime \prime \prime}$. В алгебре $\mathscr{A}(\mathscr{D})$ имеется инволюция $f \rightarrow \bar{f}$ (переход к обычной комплексной черте) и единица $e=e(\xi)$, равная 1 при $\xi=\varnothing$ и нулю при $\xi \neq \varnothing$.

Мы будем рассматривать спектральные представления позитивных функционалов $r$ в $\mathscr{A}(\mathscr{D})$, т. е. таких линейных непрерывных функционалов $\mathscr{A}(\mathscr{D}) \ni f \mapsto$ $r(f) \in \mathbb{C}$, для которых $r(f \star \bar{f}) \geqslant 0, f \in \mathscr{A}(\mathscr{D})$. В соответствии с ГНС конструкцией введем в $\mathscr{A}(\mathscr{D})$ квазискалярное произведение $(f, g):=r(f \star \bar{g}), f, g \in \mathscr{A}(\mathscr{D})$. Оно порождает соответствующее гильбертово пространство $\mathscr{H}_{r}$, строящееся как пополнение классов $\{f\}=\left\{f^{\prime} \in \mathscr{A}(\mathscr{D}) \mid\left(f-f^{\prime}, f-f^{\prime}\right)=0\right\}$.

Представление будет получено при помощи спектральной теории семейства коммутирующих самосопряженных операторов $\widetilde{A}(\varphi)$, действующих в пространстве $\mathscr{H}_{r}$ и порожденных следующей операцией в алгебре $\mathscr{A}(\mathscr{D}): \mathscr{A}(\mathscr{D}) \ni f \mapsto$ $\varphi \star f \in \mathscr{A}(\mathscr{D}), \varphi \in \mathscr{D}$. Из вида $(\cdot, \cdot)$ следует, что эта операция эрмитова, поэтому сохраняет классы и может быть записана как оператор $A(\varphi)$ в пространстве классов $\{\mathscr{A}(\mathscr{D})\} \subset \mathscr{H}_{r}$. Операторы $A(\varphi), \varphi \in \mathscr{D}$, эрмитовы, вещественны относительно введенной инволюции, линейно зависят от параметра $\varphi$ и формально коммутируют.

Известно [9], что их замыкания $\widetilde{A}(\varphi)$ в пространстве $\mathscr{H}_{r}$ будут самосопряженными, коммутирующими, линейно зависящими от $\varphi$ и вещественными операторами, если выполнено следующее предположение: (i) cyществует множество $D \subset$ $\{\mathscr{A}(\mathscr{D})\}$, инвариантное относительно действия операторов $A(\varphi)$, тотальное в $\mathscr{H}_{r}$ и такое, что любой вектор $\{f\} \in D$ будет квазианалитическим для каждого оператора $A(\varphi)$ (т.е. класс $C\left\{m_{n}\right\}$, где $m_{n}=\left\|(A(\varphi))^{n}\{f\}\right\|_{\mathscr{H}_{r}}$, $n \in \mathbb{N}_{0}$, квазианалитический).

Предположение (i) будем считать выполненным. В ряде случаев можно выписать достаточные условия на функционал $r$, обеспечивающие выполнение (i). 
Мы приведем такое условие в важном случае, когда $r$ порожден $\sigma$-конечной борелевской мерой на $\ddot{\Gamma}_{X, 0}$ :

$$
r(f)=\int_{\ddot{\Gamma}_{X, 0}} f(\xi) d \rho(\xi), \quad f \in \mathscr{A}(\mathscr{D})=\mathscr{F}_{\text {fin }}(\mathscr{D}) .
$$

Теорема 1. Для позитивного функционала вида (4) условие (i) будет выполнено, если для каждых компакта $\Lambda \subset X u k \in \mathbb{N}$ будет квазианалитическим класс $C\left\{m_{n}\right\}$, где

$$
m_{n}=\left(\sum_{l=k}^{2 k} \sum_{j=0}^{2 n} \frac{(l+j-1) !}{(l-1) !} \rho\left(\ddot{\Gamma}_{\Lambda}^{(l+j)}\right)\right)^{1 / 2}, \quad n \in \mathbb{N}_{0} .
$$

Эта теорема содержит некоторые более обозримые условия выполнения предположения (i). Так, например, (i) будет выполнено, если для каждого компакта $\Lambda \subset X$ существует константа $C_{\Lambda}>0$, такая, ито $\rho\left(\ddot{\Gamma}_{\Lambda}^{(n)}\right) \leqslant C_{\Lambda}^{n}, n \in \mathbb{N}$.

3. Построение разложения по совместным обобщенным собственным векторам (с.о.с.в.) коммутирующего семейства самосопряженных операторов $A=$ $(\widetilde{A}(\varphi))_{\varphi \in \mathscr{D}}$ в пространстве $\mathscr{H}_{r}$, линейно зависящих от параметра $\varphi$, ведется согласно схеме книги [9].

Согласно этой схеме, требуется построить гильбертово оснащение пространства $\mathscr{H}_{r}$, стандартно связанное с семейством $A$. Его построение ведется последовательно с учетом следующих моментов. 1) Пространство $\mathscr{D}$ является проективным пределом взвешенных соболевских пространств $H_{\tau}$ (по римановой мере $m$ ), где $\tau=\left(\tau_{1}, \tau_{2}(x)\right)\left(\tau_{1} \in \mathbb{N}_{0}-\right.$ порядок производных, $1 \leqslant \tau_{2}(x) \in C^{\infty}(X)-$ вес $)$. 2) Пространство $\mathscr{F}_{\text {fin }}(\mathscr{D})$ является проективным пределом взвешенных фоковских пространств $\mathscr{F}\left(H_{\tau}, p\right)$, построенных по всевозможным $H_{\tau}$ и весам $p=\left(p_{n}\right)_{n=0}^{\infty}$, $\left.p_{n} \geqslant 1.3\right)$ Функционал $r$ принадлежит $\left(\mathscr{F}_{\text {fin }}(\mathscr{D})\right)^{\prime}$ и поэтому непрерывен на некотором $\mathscr{F}\left(H_{\tau}, p\right)$. 4) Свертка $\star$ непрерывна в $\mathscr{F}_{\text {fin }}(\mathscr{D})$ и поэтому действует непрерывно в шкале пространств $\mathscr{F}\left(H_{\tau}, p\right)$; квазискалярное произведение $(f, g)$ определено для $f, g \in \mathscr{F}\left(H_{\tau^{\prime}}, p^{\prime}\right)$ при некоторых $\tau^{\prime}$ и $\left.p^{\prime} .5\right)$ Выбирая теперь $\tau^{0}$ и $p^{0}$ «достаточно большими» по отношению к $\tau^{\prime}$ и $p^{\prime}$, можно добиться, чтобы в цепочке с нулевым пространством $\mathscr{H}_{r}$

$$
\left\{\mathscr{F}\left(H_{\tau^{0}}, p^{0}\right)\right\}_{-} \supset \mathscr{H}_{r} \supset\left\{\mathscr{F}\left(H_{\tau^{0}}, p^{0}\right)\right\} \supset\{\mathscr{A}(\mathscr{D})\}
$$

вложение позитивного пространства в $\mathscr{H}_{r}$ было квазиядерным; цепочка (5) основная для построения разложения. 6) Наряду с (5) рассмотрим цепочку

$$
\left(\mathscr{F}\left(H_{\tau^{0}}, p^{0}\right)\right)_{-} \supset \mathscr{F}(H)=\bigoplus_{n=1}^{\infty} \mathscr{F}_{n}(H) \supset \mathscr{F}\left(H_{\tau^{0}}, p^{0}\right),
$$

где $\mathscr{F}(H)$ - обычное фоковское пространство, построенное по $H=L^{2}(X, d m(x))$; так как в (5) и (6) позитивные пространства совпадают (с точностью до образования классов), то негативные пространства будут изоморфными [1]. 7) Последнее позволяет с.о.с.в. семейства $A$ понимать как векторы из $\left(\mathscr{F}\left(H_{\tau^{0}}, p^{0}\right)\right)_{-}$.

Отметим также, что, как следует из $(3)$, оператор $A(\varphi)$ порождается оператором $a_{+}(\varphi)+a_{0}(\varphi), \varphi \in \mathscr{D}$, где $a_{+}(\varphi)$ и $a_{0}(\varphi)-$ классические оператор рождения 
и нейтральный оператор в $\mathscr{F}(H)$. Это дает возможность доказать наличие сильного циклического вектора для семейства $A$ и подсчитать с.о.с.в. для $A$. Мы наметили доказательство следующего результата.

Теорема 2. Семейство $A=(\widetilde{A}(\varphi))_{\varphi \in \mathscr{D}}$ порождает преобразование Фурье $I$ при разложении по его с.о.с.в.:

$$
\begin{aligned}
\mathscr{F}_{\text {fin }}(\mathscr{D}) \ni f & =\left(f_{n}\right)_{n=0}^{\infty} \mapsto(I f)(\omega)=: \hat{f}(\omega) \\
& =\sum_{n=0}^{\infty}\left(f_{n}, P_{n}(\omega)\right)_{\mathscr{F}_{n}(H)} \in L^{2}\left(\mathscr{D}^{\prime}, d \mu(\omega)\right) .
\end{aligned}
$$

Здесь $P(\omega)=\left(P_{n}(\omega)\right)_{n=0}^{\infty}, P_{n}(\omega) \in\left(\mathscr{D}^{\prime}\right)^{\widehat{\otimes} n}$, - с.о.с.в. семейства $A$, отвечающий «собственному числу» $\omega \in \mathscr{D}^{\prime}, \mathscr{D}^{\prime}-$ классическое пространство обобщенньх функиции и н-борелевская вероятностная мера на $\mathscr{D}^{\prime}$ (спектральная мера семейства $A)$.

Oператор Фурье I после замыкания по непрерывности является унитарным оператором, переводящим пространство $\mathscr{H}_{r}$ в $L^{2}\left(\mathscr{D}^{\prime}, d \mu(\omega)\right)$; при этом образом оператора $\widetilde{A}(\varphi)$ будет оператор умножения на функцию $(\omega, \varphi)_{H}$. Равенство Парсеваля сейчас имеет вид

$$
(f, g)_{\mathscr{H}_{r}}=r(f \star \bar{g})=\int_{\mathscr{D}^{\prime}} \hat{f}(\omega) \overline{\hat{g}(\omega)} d \mu(\omega), \quad f, g \in \mathscr{F}_{\text {fin }}(\mathscr{D}) \text {. }
$$

Векторы $P_{n}(\omega)$ удовлетворяют следующему рекуррентному соотношению, c помощью которого они могут быть подсчитаны:

$$
\begin{gathered}
\left(P_{n+1}(\omega), \varphi^{\otimes(n+1)}\right)_{\mathscr{F}_{n+1}(H)} \\
=\frac{1}{n+1}\left[\left(P_{n}(\omega) \widehat{\otimes} \omega, \varphi^{\otimes(n+1)}\right)_{\mathscr{F}_{n+1}(H)}-\left(P_{n}(\omega), n \varphi^{2} \widehat{\otimes} \varphi^{\otimes(n-1)}\right)_{\mathscr{F}_{n}(H)}\right], \\
n \in \mathbb{N}_{0}, \varphi \in \mathscr{D}, P_{0}(\omega)=1, P_{1}(\omega)=\omega .
\end{gathered}
$$

Полагая в (8) $g=e$ и учитывая $(7)$, получаем

$$
\begin{aligned}
r(f) & =\int_{\mathscr{D}^{\prime}}(I f)(\omega) d \mu(\omega), & f \in \mathscr{F}_{\text {fin }}(\mathscr{D}), \\
r\left(f_{n}\right) & =\int_{\mathscr{D}^{\prime}}\left(f_{n}, P_{n}(\omega)\right)_{\mathscr{F}_{n}(H)} d \mu(\omega), & f_{n} \in \mathscr{D}_{c}^{\widehat{\otimes} n}, n \in \mathbb{N}_{0} .
\end{aligned}
$$

Последняя формула решает рассматриваемую проблему моментов: позитивный функционал $r$ при выполнении условия (i) имеет представление (10); обратно, функционалы вида (10) будут позитивными.

Первая из формул (10) показывает, что в понятном смысле $r=I^{*} \mu$ и $r$ можно воспринимать как «корреляционный функционал» меры $\mu$. Тогда приведенный результат дает достаточное условие того, что функционал $r \in\left(\mathscr{F}_{\text {fin }}(\mathscr{D})\right)^{\prime}$ будет корреляционным.

4. Остановимся на случае функционала $r$, порожденного по формуле (4) мерой $\rho$ или зарядом на $\ddot{\Gamma}_{X, 0}$ (т.е. вещественнозначной $\sigma$-конечной борелевской мерой на $\left.\ddot{\Gamma}_{X, 0}\right)$. Будем говорить, что $\omega \in \mathscr{D}^{\prime}$ порожден зарядом, если $\omega(\varphi)=\int_{X} \varphi(x) d \sigma_{\omega}(x), \varphi \in \mathscr{D}$, где $\sigma_{\omega}-$ некоторый заряд на $X$. 
Теорема 3. Пусть позитивный функционал $r$ порожден мерой $\rho$ на $\ddot{\Gamma}_{X, 0}$. Тогда для спектральной меры $\mu$ в представлении (10) множество тех $\omega \in \mathscr{D}^{\prime}$, которые порождены зарядами на $X$, имеет полную меру. Обратно, пусть указанное множество имеет полную меру. Тогда позитивный функционал порожден зарядом на $\ddot{\Gamma}_{X, 0}$.

Более того, пусть позитивный функционал $r$ на $\mathscr{F}_{\text {fin }}(\mathscr{D})$ таков, ито его сужение на $\mathscr{F}_{1}(\mathscr{D}) \subset \mathscr{F}_{\text {fin }}(\mathscr{D})$ порождено мерой на $X$. Тогда весь функиионал $r$ порожден зарядом на $\ddot{\Gamma}_{X, 0}$.

5. Перейдем к рассмотрению аналогичных вопросов в случае пространства $\Gamma_{X, 0}$ обычных конфигураций. Оно определяется как дизъюнктная сумма $\Gamma_{X, 0}:=$ $\bigsqcup_{n=0}^{\infty} \Gamma_{X}^{(n)}$, где $\Gamma_{X}^{(n)}$ лежит в $\ddot{\Gamma}_{X}^{(n)}$ и состоит из точек $\xi_{n}=\left[x_{1}, \ldots, x_{n}\right]$, в которых все $x_{j}$ различны. Таким образом, $\Gamma_{X, 0} \subset \ddot{\Gamma}_{X, 0}$ и топология пространства $\ddot{\Gamma}_{X, 0}$ индуцирует в $\Gamma_{X, 0}$ относительную топологию. Аналогичные объекты вводятся с заменой $X$ на замкнутое множество $\Lambda \subset X$ (в частности, $\left.\Gamma_{\Lambda}^{(n)}\right)$.

Нам понадобится также пространство $\Gamma_{X}$ бесконечных конфигураций, т.е. множеств $\gamma=\left[x_{1}, x_{2}, \ldots\right]$, где $x_{j}-$ различные точки из $X$, причем в каждом компакте $\Lambda \subset X$ имеется лишь конечное число этих точек; $\Gamma_{X} \supset \Gamma_{X}^{(n)}$, $n \in \mathbb{N}$. Каждую такую конфигурацию $\gamma$ можно отождествить с $\sigma$-конечной мерой $\sum_{j=0}^{\infty} \delta_{x_{j}}\left(\delta_{x}-\right.$ дельта-функция, сосредоточенная в $\left.x\right)$ и тем самым с функционалом $\gamma \in \mathscr{D}^{\prime}$. Таким образом, $\Gamma_{X} \subset \mathscr{D}^{\prime}$ и $\mathscr{D}^{\prime}$ индуцирует на $\Gamma_{X}$ относительную топологию.

Введем отображение $K$, переводящее функции из $\mathscr{F}_{\text {fin }}(\mathscr{D})$ в функции на $\Gamma_{X}$, по формуле

$$
\mathscr{F}_{\text {fin }}(\mathscr{D}) \ni f \mapsto(K f)(\gamma)=\sum_{\xi \subset \gamma} f(\xi) \in \mathbb{C}, \quad \gamma \in \Gamma_{X}
$$

(в (11) сумма для каждой $f$ конечна). Оказывается, что при некоторых ограничениях на меру $\rho$, порождающую, согласно (4), позитивный функционал $r$, оператор Фурье из (7), (8), (10) совпадает с оператором $K$ из (11):

Теорема 4. Пусть мера $\rho$ в (4) сосредоточена на пространстве обычных конфигураций, т.е. $\rho\left(\ddot{\Gamma}_{X, 0} \backslash \Gamma_{X, 0}\right)=0$, и удовлетворяет следующей оценке: для каждого компакта $\Lambda \subset X$ существуют $C_{\Lambda}, \varepsilon_{\Lambda}>0$, такие, ито

$$
\rho\left(\Gamma_{\Lambda}^{(n)}\right) \leqslant C_{\Lambda}\left(2+\varepsilon_{\Lambda}\right)^{-n}, \quad n \in \mathbb{N}_{0} .
$$

Тогда пространство $\Gamma_{X} \subset \mathscr{D}^{\prime}$ имеет полную спектральную меру $\mu, I=K$ и $\mathscr{D}^{\prime}$ в формулах (8), (10) можно заменить на $\Gamma_{X}$.

Отметим, что оценка (12) по существу совпадает с аналогичной оценкой из [3]; представляется, что (12) может быть ослаблена. При доказательстве теоремы существенную роль играет то обстоятельство, что в общем случае теоремы 2 может быть подсчитано преобразование Фурье (7) вектора $f=\left(\varphi^{\otimes n}\right)_{n=0}^{\infty}, \varphi \in \mathscr{D}$. Оказывается, $\hat{f}(\omega)=\exp (\omega, \log (1+\varphi))_{H}$ при $\sup _{x \in X}|\varphi(x)|<1$.

В предположениях этой теоремы корреляционный функционал $r$ совпадает с обычной корреляционной мерой $\rho$ меры $\mu$, заданной на пространстве бесконечных конфигураций. 


\title{
ЛИТЕРАТУРА
}

1. Berezansky Yu. M. Integral Equations Operator Theory, 44, 255-289 (2002). 2. Kondratiev Yu. G., Kuna T. Infin. Dimens. Anal. Quantum Probab. Relat. Top., 5, No. 2, 201233 (2002) (препринт: Bonn University, 1999). 3. Berezansky Yu. M., Kondratiev Yu. G., Kuna T., Lytuynov E. Methods Funct. Anal. Topology, 5, No. 4, 87-100 (1999). 4. Гельфанд И. М., Костюченко А. Г. ДАН СССР, 103, No. 3, 349-352 (1955). 5. Березанский Ю. М. ДАН СССР, 108, №3, 379-382 (1956). 6. Березанский Ю. М. ДАН СССР, 110, №6, 893-896 (1956). 7. Березанский Ю. М. Разложение по собственным функциям самосопряженных операторов. Наукова думка, Киев, 1965. 8. Lenard A. Arch. Rational Mech. Anal., 59, 241-256 (1975). 9. Березанский Ю. М., Кондратьев Ю. Г. Спектральные методы в бесконечномерном анализе. Наукова думка, Киев, 1988.

Институт математики НАН Украины e-mail: berezan@mathber.carrier.kiev.ua

\section{О суммах проекторов в $C^{*}$-алгебрах}

\author{
(c) 2003. Т. В. ШульмАН
}

\section{Посвящается И. М. Гельфанду в связи с его 90-летием}

В работе [1] рассматривалась задача о существовании набора $p_{1}, \ldots, p_{n}$ самосопряженных проекторов в гильбертовом пространстве с суммой $\lambda 1$, где $\lambda \in \mathbb{R}$. Было получено полное описание множества $\Sigma_{n}$ всех $\lambda$, для которых такие проекторы существуют - при $n \geqslant 5$ оно представляет собой отрезок $\left[\alpha_{n}, \beta_{n}\right]$, объединенный с двумя последовательностями $S_{n}^{1}$ и $S_{n}^{2}$, сходящимися к $\alpha_{n}$ и $\beta_{n}$ соответственно. Через $\mathscr{P}_{n, \lambda}$ обозначалась универсальная $C^{*}$-алгебра этой задачи, т. е. $C^{*}$-алгебра с образующими $p_{1}, \ldots, p_{n}$ и соотношениями $p_{i}=p_{i}^{*}=p_{i}^{2}$, $\sum_{i=1}^{n} p_{i}=\lambda e$. Наборы проекторов указанного выше вида соответствуют представлениям алгебры $\mathscr{P}_{n, \lambda}$. Соответственно унитальные гомоморфизмы алгебры $\mathscr{P}_{n, \lambda}$ в произвольную унитальную $C^{*}$-алгебру $A$ характеризуются наборами проекторов из $A$, удовлетворяющих условию $\sum_{i=1}^{n} p_{i}=\lambda e$. Множество всех $\lambda$, для которых такие проекторы существуют, обозначим через $\Sigma_{n}(A)$ (таким образом, $\left.\Sigma_{n}=\Sigma_{n}(\mathscr{B}(H))\right)$.

Напомним (см. [2]), что $C^{*}$-алгебра называется алгеброй типа I, если ее образ при любом неприводимом представлении содержит все компактные операторы в пространстве представления. В [1] было доказано, что $\mathscr{P}_{n, \lambda}$ является $C^{*}$-алгеброй типа I при $\lambda \in S_{n}^{1}, \lambda \in S_{n}^{2}$ и не является алгеброй типа I при $\lambda \in\left(\alpha_{n}, \beta_{n}\right)$, и был поставлен вопрос, имеет ли $\mathscr{P}_{n, \lambda}$ тип I при $\lambda=\alpha_{n}$ и $\lambda=\beta_{n}$. Мы даем ответ на этот вопрос в п. 1, получив общее описание множества $\Sigma_{n}(A)$ для $C^{*}$-алгебр типа I. Описание множества $\Sigma_{n}(A)$ для AF-алгебр в п. 2 дает ответ на другой вопрос авторов работы [1]: для всякой ли $C^{*}$-алгебры $A$ множество $\Sigma_{n}(A)$ замкнуто?

1. Теорема 1. Eсли $A$ есть $C^{*}$-алгебра muna I, mo $\Sigma_{n}(A)$ cocmoum из конечного множества рацииональных чисел.

Так как $\lambda \in \Sigma_{n}\left(\mathscr{P}_{n, \lambda}\right)$, то мы получаем 